\title{
An Insight into the Immunization Coverage for Combined Vaccines in Albania
}

\author{
Eftiola Pojani, PhD Student \\ Department of Pharmaceutical Sciences \\ Catholic University “Our Lady of Good Counsel” Tirana, Albania \\ Erida Nelaj, PhD \\ Department of Epidemiology and Infectious Disease Control \\ Institute of Public Health, Tirana, Albania \\ Alban Ylli, Associate Prof. \\ University of Medicine, Faculty of Public Health, Tirana, Albania \\ doi: 10.19044/esj.2016.v13n3p33 URL:http://dx.doi.org/10.19044/esj.2016.v13n3p33
}

\begin{abstract}
We aim to Provide facts about the vaccination coverage for combined vaccines such as MMR (Measles, Mumps and Rubella) and DTP (Diphtheria, Tetanus, Pertussis) containing vaccines in use in Albania during the last 10 years, in order to confirm the stability of immunization program. One of the reasons for coverage improvement is the use of one or two-dose vials for the administration of MMR vaccine, enabling the vaccination of children at any time, without the need of gathering them on certain days when the multi-dose vaccine vials are opened. In the last three years, according to WHO recommendations, it was noted that vaccination coverage with three doses containing DTP is at sustainable levels, always above 95\%, but for our country this value goes sometimes above $98 \%$. As for the use of pentavalent vaccine, from 2008, DTP-HepB-Hib, also the coverage of Hepatitis b vaccine is always at upper levels due to its use on 5 in 1 combination. The application of this vaccine was associated with the use of one dose vial administration, therefore one vaccine to a child. Immunization coverage for vaccines with measles component remains at very high levels for both doses of vaccine, more then 95\%. Immunization coverage for vaccines with DTP component exceeds 95\% rate at country level, for each district it exceeds $95 \%$ and it reaches the value of $98.5 \%$ for the last two years. During the last years the vaccine coverage is increased significantly as a result of using combined vaccine of DTP-HepB -Hib. The use of one dose vials has played an important role on sustaininig and increasing vaccination coverage.
\end{abstract}


Keywords: MMR, DTP, combined vaccine, vaccination coverage

\section{Introduction}

In Albania, the measles vaccine has been in regular use since 1971 as a single antigen. Due to the epidemic outbreak of 1970-71, a mass vaccination, with the imported B55 vaccine, was urgently taken for the first time in Albania, for all groups of children born in the period 1956-1970. In 1971 induced routine vaccination against measles was introduced in the national immunization schedule for all new births that would follow and in 1977 measles vaccine began to be produced even in the country.

In 1992, domestic production of B55 vaccine was interrupted, being replaced by the imported Schwarz vaccine. Since January 200, Albania has introduced the bi-valent measles and rubella vaccine in the compulsory vaccination calendar (Kakarriqi, 2002) giving the child a basic dose on the 12th month of birth and the booster dose at age of 5. Mumps component was added to the National Immunization Program since January 2005 with the introduction of the three-valent vaccine, which is still in use in Albania today.

Measles is considered as a disease that could disappear because of a single serotype, effective vaccine, lack of human natural reservoirs and high clinical expression of the disease. For years an attempt has been made to eliminate measles through continuous vaccination to prevent its recurrence achieving so the "herd" immunity. Global eradication will be based on the successful elimination in all countries.

The most recent assessment by WHO for measles vaccine coverage (Figure 1) shows that globally the values are over $90 \%$ mostly in American and European regions. Much work remains to be done in order to increase vaccine coverage in the African region and partly in the Asian one (WHO, 2016).

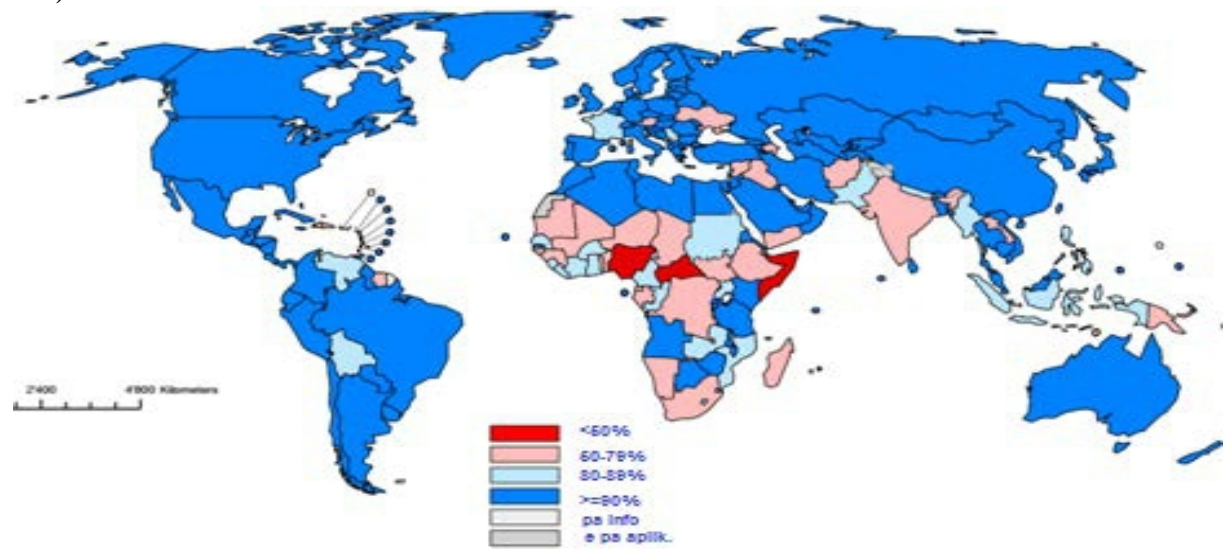

Figure 1. Global Immunization coverage for vaccines containing measles Source: (WHO / UNICEF, 2013) 
Regarding tetanus, diphtheria and pertussis are diseases that might be very serious and caused by bacteria. Tetanus enters the body through the cracks, deep scratches and wounds, while diphtheria and pertussis are spread from person to person through coughing or sneezing. (CDC, 2012)

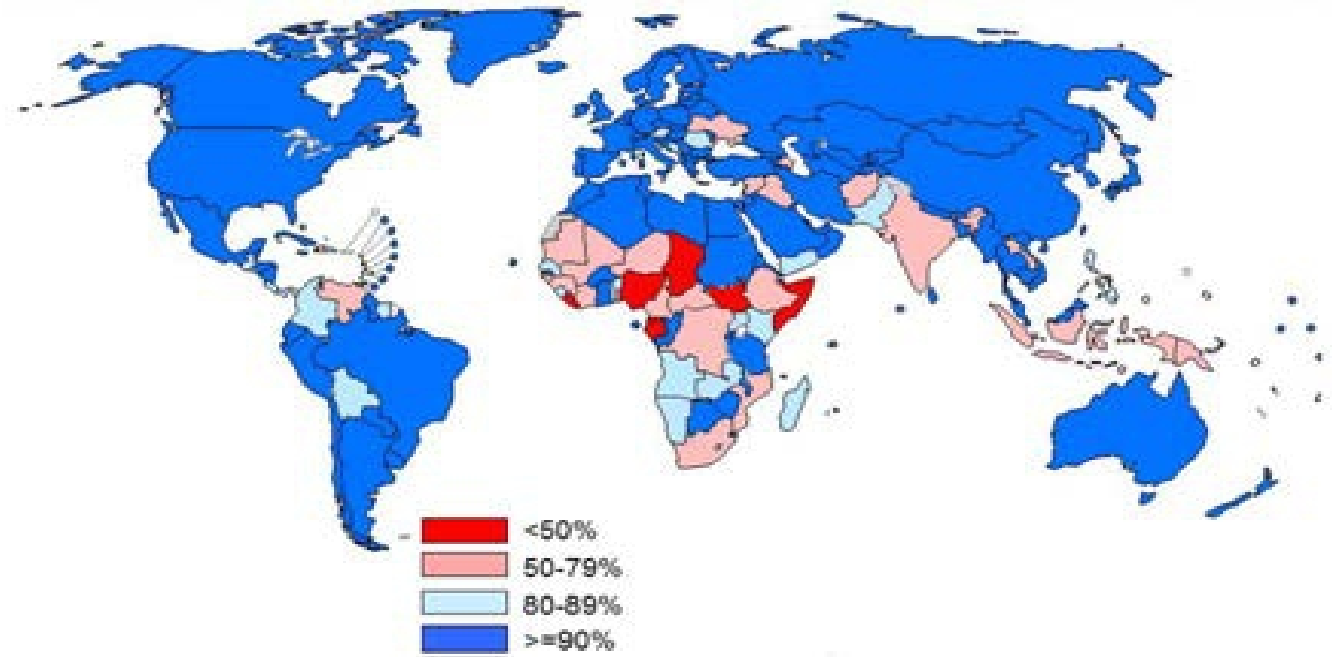

Figure 2. DTP3 vaccine coverage (diphtheria, tetanus anatoksina and pertus, with three doses) in children, 2011. Source (WHO database, 2012)

\section{Materials and Methods}

Routine levels of vaccination coverage indicate the vaccination program performance and provide an overview of immunity in the population. Immunization coverage is usually assessed on the basis of the percentage of children who have received a certain number of recommended vaccine dose during the first year of life. In the same way, the vaccination coverage is also evaluated for the recommended vaccines doses for revaccination (reminder immune). This vaccination coverage is nothing but a report of the number of children vaccinated versus the number of children scheduled for vaccination. The reported covarage data taken from the vaccination centers are aggregated to higher levels by providing values at the district, prefecture and national levels. (WHO, 2010)

In this study, we tried to provide facts about the vaccine coverage for combined vaccines such as MMR and DTP containing vaccines in use in Albania during the last 10 years, in order to confirm the stability of immunization program.

The percentage of vaccine coverage for each vaccination (immunization) indicator in all districts of the country during the years 20032013, for each antigen obtained in the study.

Immunization coverage in percentage is calculated by the formula: 


$$
\begin{aligned}
& \text { Immunization coverage }(\%) \\
& =\frac{\text { The number of vaccinated children }}{\text { Number of children scheduled for vaccination }} \times 100
\end{aligned}
$$

\section{Results:}

In the context of eliminating measles (Kakarriqi and Bino, 2002), an important factor for vaccine coverage is its level over $95 \%$ at country level and at the district level over $90 \%$, or vaccination coverage $90 \%$ for two doses of vaccine containing component of measles, for all children until 2015.

MR vaccine was used in the years 2003-2004 and then, from 2005 until now the MMR vaccine has been used in the national immunization calendar (Bino et al, 2003).

Graphically this coverage is presented in the following graph (1) where it is obvious that this coverage for both doses exceeds levels of $95 \%$, except for 2006, during which the national program of vaccination was faced with a shortage of this vaccine globally and exactly in this year two measles outbreaks occurred, mainly in the Roma population of Saranda and Elbasan districts.

Furthermore, a reason for the improvement of this coverage is the use of one dose bottles administration for MMR vaccine, enabling the vaccination of children at any time, without the need of collecting them on certain days when the vaccine bottles are opened.

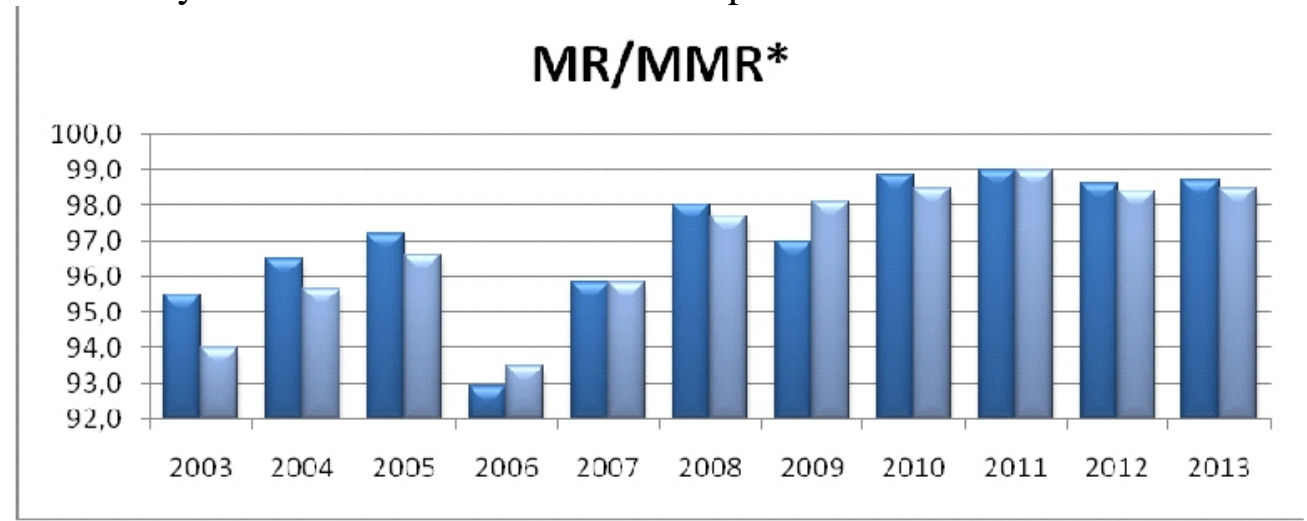

Graph 1. Immunization coverage with measles vaccines in years.* Mumps component (parotit) was introduced in 2005

Although in the global strategy for the elimination of measles (Hall and Jolley, 2011) the vaccination coverage in every district should be above $90 \%$, for our country it is clearly seen that this coverage is in very high levels, i.e. well above 95\%. For the first dose the average value is $98.8 \%$ and with IC [98.4\% -99.3\%], which shows that vaccination coverage is also high at the district level. (Graph.1) 
It is worth mentioning that since 2009 the hepatitis B component was included in the combined pentavalent DTP-HepB-Hib vaccine.

As it is seen even graphically, this coverage is above $95 \%$ of the value according to WHO recommendations (WHO, 2014), but for our country, the average value is $98.3 \%$ with IC [98.0\% $-98.5 \%$ ], which indicates a very high level of coverage with this vaccine, already combined 5 in 1.

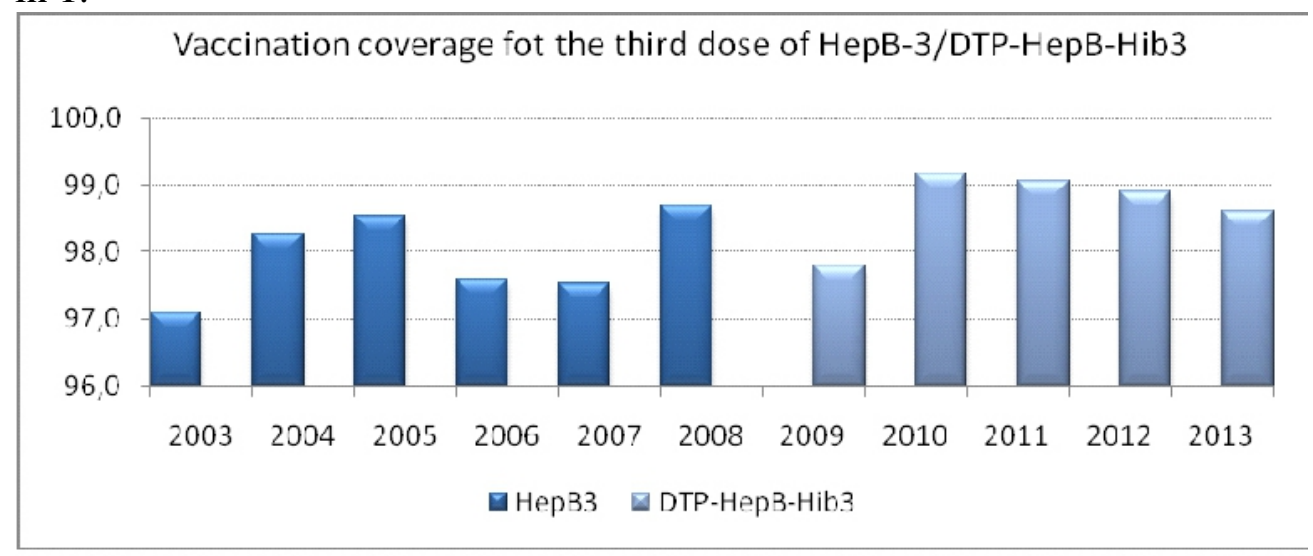

Graph 2. Vaccination coverage for the third dose of hepatitis B, 2003-2013

Especially considering the last three years, it is noted that vaccination coverage with three doses of vaccine containing hepatitis B is at sustainable levels (Nelaj et al, 2014) always above 95\%, according to the recommendations of the WHO, but for our country this value reaches above $98 \%$. One of the factors of reaching high levels is the use of the combined vaccine 5 in 1, enabling child vaccination with only one injection. The other reason is that since 2008, the introduction of this vaccine was associated with its use in one-vial dose administration, that ia to say, a vaccine to a child. Some years ago, mainly in the rural areas, vaccination centers used the practice of approximate date of vaccination by assembling children for vaccination in one or two days a month, so there was no significant loss in the use of the multi-dose bottle; while by using one-dose bottle vaccines the child is vaccinated almost exactly on the appointed day, with rare exceptions, when vaccination is postponed for health reasons.

Graphical presentation clearly demonstrates the viability of the vaccine in very high levels. 


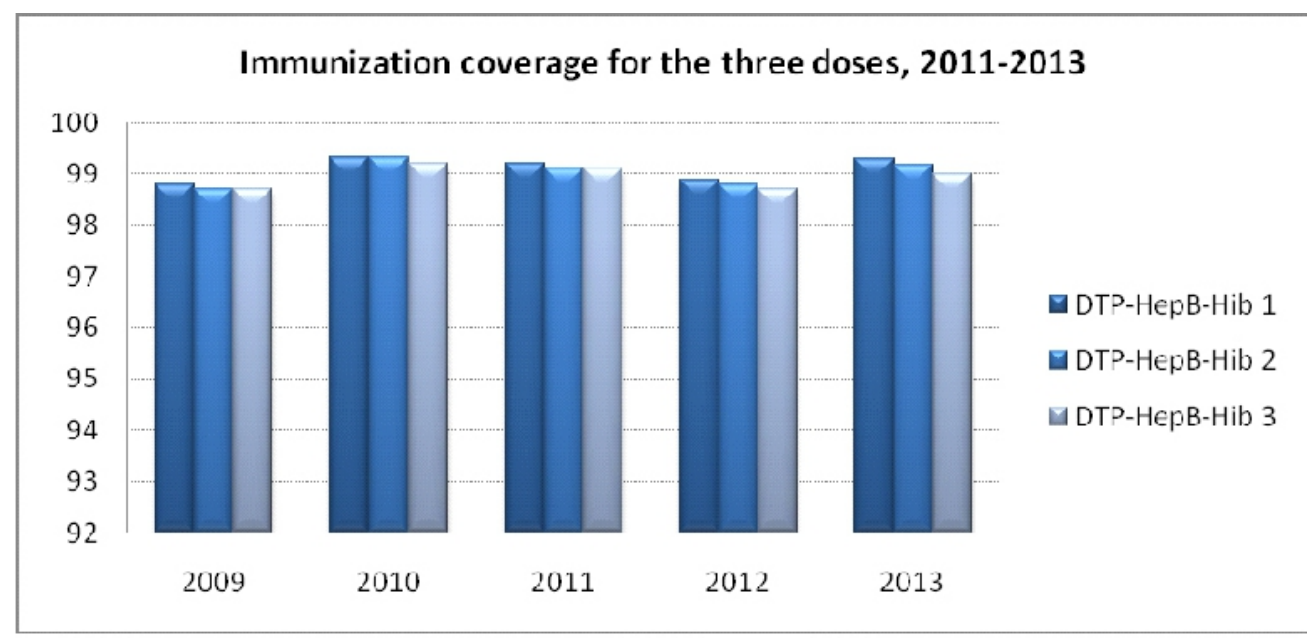

Graph 3. Immunization coverage for the three doses of DTP-HepB-Hib, in 2011-2013

Graph 3 clearly shows that high levels of this coverage are reflected by the very high coverage in each district of the country, where the coverage values go beyond the $98 \%$. Specifically the average value is $99.2 \%$ with IC [98.9\% -99.5\%].

\section{DTP vaccination coverage for Albania}

As it was mentioned in the previous evaluation of the coverage for other vaccine antigens, in the immunization global strategy (WHO,2016), the important factor for vaccine coverage is its value over 95\% at the national level and at the district level above $90 \%$ for the three vaccine doses containing diphtheria, tetanus and pertus.

For the evaluation of vaccine coverage, the application of the third dose with DTP component is taken into consideration, since it means the fulfillment of a series of vaccination with this vaccine, according to the national immunization schedule. Immunization coverage for three applied vaccine doses with DTP component for the years 2003-2013 appears to be above $95 \%$ at the country and district level. As it was mentined, since 2009, DTP components were included in the pentavalent DTP-HepB-Hib combined vaccine.

It is clearly seen that the level of vaccine coverage for these components (Graph 4) exceeds the value of 95\%, reaching in some cases $99 \%$ values. The average value turns out to be $98.3 \%$ 


\section{DTP3; DTP-HepB-Hib3}

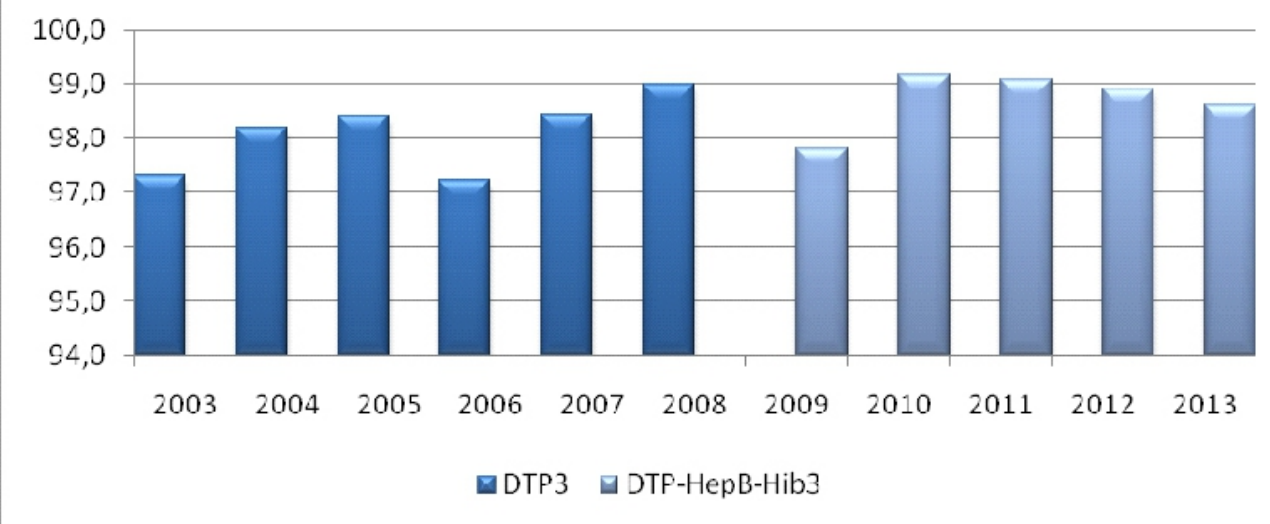

Graph 4. Immunization coverage for DTP components, 2003-2013

According to the calendar, at the two-month age the revaccination with DTP vaccine (diphtheria tetanus, pertus) is applied. Graph 5 shows the values of DTP vaccine coverage for the first booster dose. It is obviously seen that at the age of 2 vaccination with this vaccine retains (maintains) the same trend, i.e. vaccination coverage, not just $95 \%$ but more than $97 \%$.

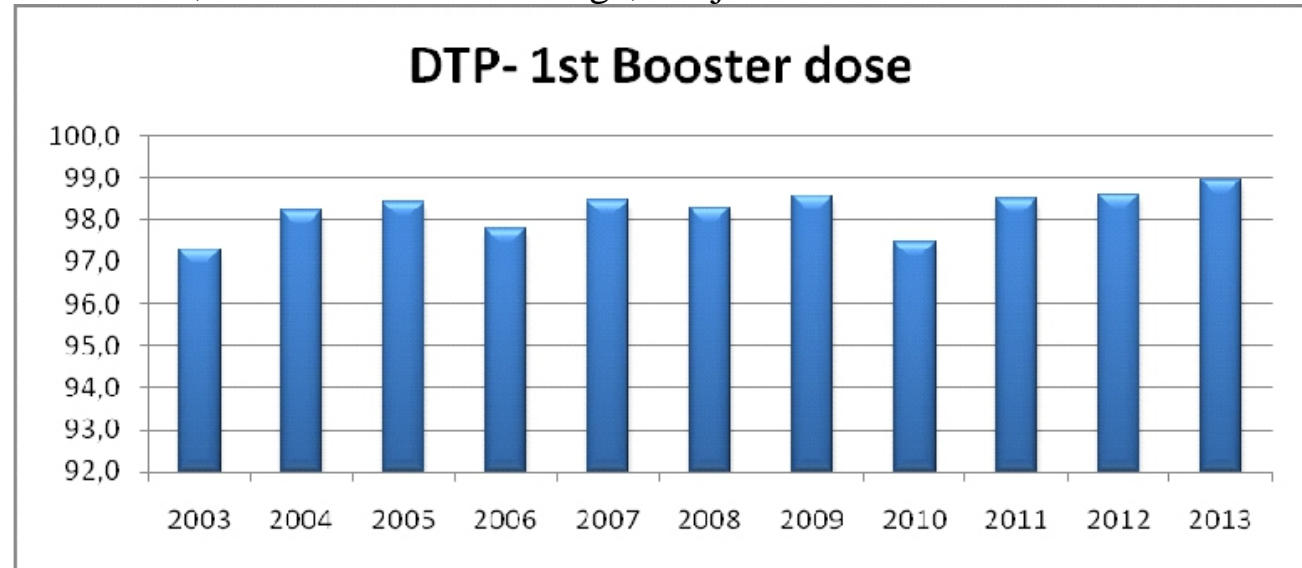

Graph 5. DTP vaccine coverage, in the first booster dose, during the years 2003-2013

At the age of 5, according to the immunization calendar, the second booster dose with DT (diphtheria tetanus) vaccine is defined 


\section{DT- 2nd Booster dose}

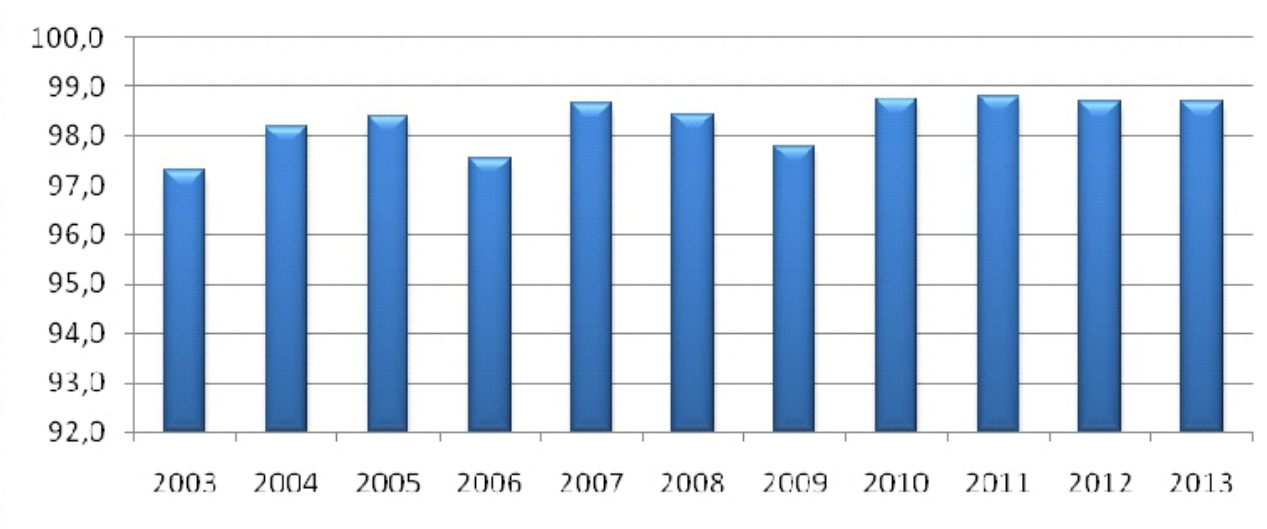

Graph 6. Immunization coverage for DT in the second booster dose, during the years 20032013

Graph 6 shows the immuniztion coverage for DT in the second booster dose. Vaccination in this age retains again the same trend, more than $97 \%$. One strong reason for maintaining these levels is the fact that children ought to be vaccinated before staring the primary school; they are strongly obliged to present their immunization records before their enrollment in schools.

At the age of 14 , as marked in the calendar, the third booster dose (tetanus, with less diphtheria) happens. Graph 7 shows the values of Immunization coverage for $\mathrm{Td}$ in the third booster dose, during the years 2003-2013

\section{Td- 3rd booster dose}

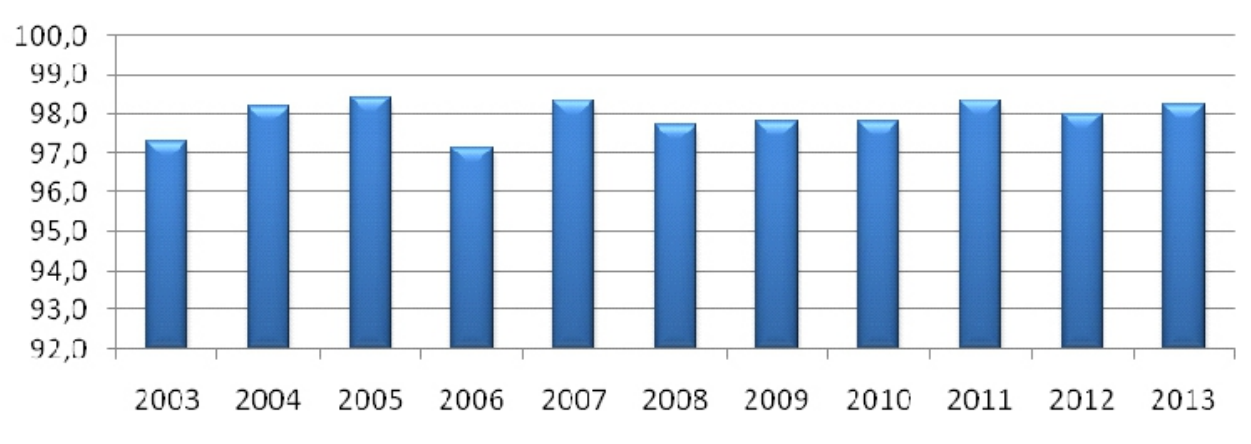

Graph 7. Immunization coverage for Td in the third booster dose, during the years 20032013 


\section{Conclusion}

Immunization coverage for vaccines with measles component remains at very high levels for both doses of vaccination.

To continue with the same high levels of vaccination coverage for MMR component vaccine, these steps should be followed: closely tracking and monitoring the vaccination calendar with the two relevant doses of MMR vaccine to all children in Albania, strengthening of measles surveillance in order to prevent any possible transmission of the disease and working to eliminate the receptive pockets, i.e. vaccination of children who are still unvaccinated due to parents’ negligence or refusal.

Immunization coverage for vaccines with DTP component exceeds $95 \%$ in the national level, for each district it is above 95\% and in the last two years, it overpasses the 95\% value. During the last years, the vaccine coverage has increased significantly as the result of using DTP-HepB-Hib pentavelente vaccine. Immunization coverage for Diphtheria-tetanus-pertus resulted to be in very high levels, above $95 \%$ and in some cases above $98 \%$ values.

Regarding tetanus booster doses, it is recommended that this calendar will not only cover children aged 0-14 years, but also adults and the elderly ones. In addition, ways of reporting coverage vaccination should also include children with backward vaccination.

\section{References:}

1. Bino S, Kakarriqi E, Xibinaku M, Ion-Nedelcu N, Bukli M, Emiroglu $\mathrm{N}$, et al. (2003). Measles-rubella mass immunization campaign in Albania;187 (Suppl 1):S223-9.

2. CDC-Course Textbook Epidemiology and Prevention of VaccinePreventable Diseases (May 2012) The Pink Book:, 12th (Ed) Printing- Hepatitis B module 115-138; Measles module (pp173-193); Tetanus module (pp 77-85); Diphtheria module (pp 86-95); Pertusis module (pp 215-232).

3. Centers for Disease Control and Prevention. Epidemiology and Prevention of Vaccine-Preventable Diseases.The Pink Book: 13th Edition (2015)

4. Eduard Z. Kakarriqi, Silva Bino. Shqipëria në fazën e eliminimit të Fruthit (Periudha 2001 e pas) Survejanca epidemiologjike e fruthit dhe rubeolës. I.Sh.P 2002; (pp21-40-47).

5. Kakarriqi,E. (2002). Epidemiological background of infectious diseases in Albania 1960-2001 and their prevention and control in the context of natural disasters and infectious diseases. Tirana, Albania. 
6. Nelaj. E, Lika (Çekani) M, Bino S, (2014). Impact of hepatitis $b$ vaccination as an important indicator for immunization program in Albania. Journal of Life sciences, vol 4.

7. Robert Hall and Damien Jolley (2011). International Measles Incidence and Immunization Coverage. Journal of Infectious Diseases. 204 (suppl 1):S158-S163.doi: 10.1093/infdis/jir124

8. World Health Organization (2016). Measles immunization coverage. Global Health Observatory (GHO).

9. World Health Organization (2014). Immunization coverage reaches $84 \%$, still short of $90 \%$ goal. Immunization, Vaccines and Biologicals. WHO July 2014

10. World Health Organization (2016).Global immunization coverage sustained in the past five years. Immunization, Vaccines and Biologicals. WHO July 2016.

11. World Health Organization [WHO Immunization in practice 2010]

12. World Health Organization WHO Database 2012

13. World Health Organization WHO Database 2013 